\title{
Minireview
}

\section{Integrating BRAF/MEK inhibitors into combination therapy for} melanoma

\author{
KSM Smalley ${ }^{*, 1}$ and KT Flaherty ${ }^{2}$ \\ 'Molecular Oncology Department, The Moffitt Cancer Center and Research Institute, 12902 Magnolia Drive, Tampa, FL 336I 2, USA; ${ }^{2}$ Department of \\ Hematology-Oncology and The Abramson Cancer Center, University of Pennsylvania School of Medicine, Philadelphia, PA 19104, USA
}

The discovery of BRAF mutations in melanoma has not yet translated into clinical success, suggesting that BRAF/MEK inhibitors will need to be combined with other agents. In the current review, we discuss other pathways likely to be important for melanoma progression and suggest possible drug combinations for future clinical testing.

British Journal of Cancer (2009) I 00, 431 -435. doi: I0.1038/sj.bjc.660489I www.bjcancer.com

Published online 20 January 2009

(c) 2009 Cancer Research UK

Keywords: melanoma; targeted therapy; BRAF; PI3K; mTOR; PTEN; chemotherapy

The discovery of activating oncogenic mutations in $B R A F$ in over $60 \%$ of melanomas has raised expectations that melanoma may be amenable to targeted therapy. The most prevalent mutation in $B R A F$ is at the $\mathrm{V} 600 \mathrm{E}$ position, resulting in the substitution of valine to glutamate and destabilisation of the inactive kinase conformation switching the equilibrium towards the active form (Gray-Schopfer et al, 2007). Most of the transforming activity of the BRAF V600E is thought to result through the activation of the mitogen-activated protein kinase (MAPK) pathway (Gray-Schopfer et al, 2007). Regardless of BRAF V600E mutational status, virtually all melanomas have activity in the MAPK pathway (Satyamoorthy et al, 2003), and this contributes to the oncogenic phenotype of melanoma through its effects on cell proliferation, invasion and survival (Gray-Schopfer et al, 2007). In experimental systems, the role of $B R A F$ in melanoma seems convincing. In vitro studies have shown that BRAF V600E is an oncogene in immortalised mouse melanocytes (Gray-Schopfer et al, 2007), and the selective downregulation of $B R A F$ V600E using RNAi causes cell death and reversal of the melanoma phenotype (Hingorani et al, 2003). However, pharmacological inhibition of BRAF and MEK have not produced such dramatic effects in vivo (Sharma et al, 2005; Haass et al, 2008). This is partly due to inherent flaws in the agents tested to date, and also a likely indication of the greater vulnerability of melanoma cells in vitro where most of the cells are rapidly cycling under sub-confluent culture conditions in the continuous presence of serum.

\section{PRECLINICAL STUDIES ON BRAF/MEK INHIBITORS}

The first putative BRAF inhibitor to be developed was sorafenib (Nexavar ${ }^{\mathbb{R}}$, BAY 43-9006). A number of studies have now shown that sorafenib induces melanoma cell apoptosis in vitro and reduces the growth of human melanoma xenografts in mice

*Correspondence: Dr KSM Smalley; E-mail: Keiran.Smalley@Moffitt.org Received 7 November 2008; revised 9 December 2008; accepted I8 December 2008; published online 20 January 2009
(Sharma et al, 2005). Although it was shown that phospho-MEK was blocked at the concentrations of sorafenib used, only relatively minor levels of apoptosis were observed in vivo, suggestive of alternative mechanisms of action. It was later shown that sorafenib treatment reduced the vascularisation of melanoma xenografts (Sharma et al, 2005), and that the compound was a relatively potent VEGF receptor inhibitor.

A number of more specific BRAF inhibitors have now been developed, some of which have relative selectivity for the $B R A F$ V600E mutation compared with wild-type BRAF, such as SB590885 (GlaxoSmithKline, Collegeville, PA, USA) (King et al, 2006) and PLX-4032/PLX-4720 (Plexxikon, Berkley, CA, USA) (Tsai et al, 2008). As selective BRAF targeted compounds have relatively few off-target effects, it is now possible to assess the effects of specific pharmacological inhibition of BRAF in melanoma. An extensive characterisation of SB590885 has shown that the compound is highly selective for cell lines with $B R A F$ mutations, accompanied by a profound inhibition of cell growth associated with the induction of G1-phase cell cycle arrest (King et al, 2006). Interestingly, SB590885 activity against human melanoma xenografts in mice is fairly weak, and there is merely a delay in the onset of tumor growth. The interpretation of these results is somewhat hindered by the poor pharmacological properties of this agent. Similar G1-phase growth arrest results have been observed with allosteric MEK inhibitors, such as U0126, PD0325901 and AZD6244, suggesting that inhibition of the MAPK pathway in melanoma is largely cytostatic (Smalley et al, 2006; Solit et al, 2006; Haass et al, 2008). Activating BRAF V600E mutations are also known to occur in subsets of thyroid and colon carcinomas (Davies et al, 2002), and similar to melanoma, the presence of the mutation also predicts for sensitivity to MEK inhibition (Solit et al, 2006; Leboeuf et al, 2008). There is evidence that PLX-4032 and its tool-compound counterpart PLX-4720 induces some limited apoptosis in melanoma cell lines with the V600E mutation but not in those that are BRAF wild type (Tsai et al, 2008). Given the pivotal role of $B R A F$ in melanoma progression, it is somewhat surprising that these pharmacological inhibitors do not generally induce much apoptosis. Recent studies have shown that blocking the MAPK pathway did not affect the levels of Bcl-2, Bcl-XL 


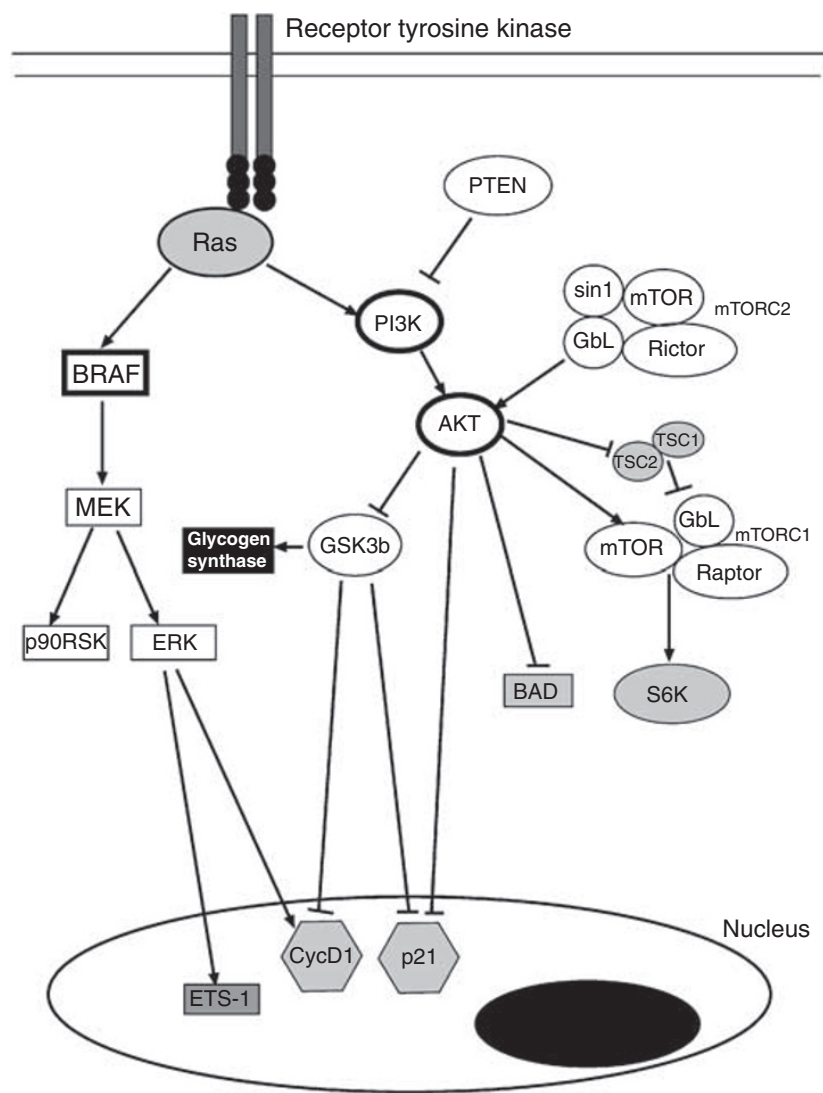

Figure I Sample signalling scheme showing pathways known to be active in melanoma. Preclinical studies support the combined targeting of either BRAF/MAPK and PI3K/AKT pathway or the BRAF/MAPK and mTOR signalling pathways in melanoma. It is likely that the combined inhibition of BRAF/PI3K pathways will have synergistic effects at the level of growth inhibition, via effects upon cyclin DI expression, as well as through increased expression of the cyclin dependent kinase inhibitor $\mathrm{p} 2$ I waf-I/cip-1. Inhibition of the AKT/mTOR pathway is likely to affect cell survival via modulation of $\mathrm{BAD} / \mathrm{BCl}-2$, as well as affecting protein translation at the level of S-6-kinase (S6K).

or Mcl-1 expression (Verhaegen et al, 2006). Knockdown of the BH3-family protein Mcl-1 using shRNA sensitised the melanoma cells to U0126-induced apoptosis, showing that overexpression of these key anti-apoptotic proteins in melanoma is a critical barrier to an effective BRAF/MEK inhibitor therapy. It is therefore suggested that melanoma cells recruit additional survival mechanisms that are MAPK pathway-independent.

\section{WHY COMBINATION THERAPIES ARE NEEDED IN MELANOMA}

It is likely that as the melanomas progress, there is functional redundancy between the numerous signalling pathways (Figure 1). Earlier studies from our laboratory, using cell lines derived from metastatic lesions, confirm this hypothesis and demonstrate that cells are resistant to both MEK and PI3K inhibitors when grown in 3D culture (Smalley et al, 2006). It was further shown that targeting either the PI3K or MEK pathway alone led to cytostasis and was associated with a reversible G1-phase cell cycle arrest (Smalley et al, 2006; Haass et al, 2008). In other studies, there was a lack of good correlation between the inhibition of either the PI3K or MEK signalling pathway and the inhibition of cell growth, showing a functional redundancy between the various growth-promoting signalling pathways. These findings also translated into in vivo studies, where the MEK inhibitor AZD6244 led to the stabilisation of established human melanoma xenografts, but not tumor regression (Haass et al, 2008). Furthermore, this suggests that the effects of MEK inhibition in this setting were largely cytostatic. This lack of good cytotoxic activity after either MEK or PI3K inhibition alone suggests that multiple signalling pathways need to be targeted simultaneously to induce melanoma regression.

It is currently unclear how well the preclinical findings on the role of $B R A F$ in melanoma cell lines match with the clinical observations on the role of $B R A F$ in melanoma pathogenesis. A number of reports have suggested that the levels of phospho-ERK staining are often variable in patient tumors and do not correlate with the BRAF/NRAS mutational status (Houben et al, 2008). Even in melanomas that are the most sensitive, prolonged BRAF/MEK inhibitor treatment may lead to the rapid acquisition of drug resistance. Earlier studies have shown that prolonged treatment (>6 months) with the MEK inhibitor CI-1040 leads to a 100-fold loss of potency in apoptosis and colony formation assays associated with increased expression of activated KRAS (Wang et al, 2005). Some of the first reports have now appeared indicating that melanoma cells can also become resistant to BRAF inhibitors after continuous drug exposure. Treatment of melanoma cells with the BRAF inhibitor AZ628 led to the development of clones that maintained high phospho-ERK levels and continued to proliferate in the presence of drug (Montagut et al, 2008). In this instance, resistance was associated with a switching from BRAF to CRAF signalling, an effect that could be reversed by treatment with the heat shock protein (HSP)-90 inhibitor geldanamycin (Montagut et al, 2008). There is also recent evidence from our group demonstrating that some melanomas with $B R A F$ V600E mutations may be intrinsically resistant to inhibitors of BRAF as a result of cyclin D1 amplification (Smalley et al, 2008).

The key question facing the field is what combination of signal transduction inhibitors are needed to achieve the maximal cytotoxic effect, and whether the combination of inhibitors is determined by the underlying genetics of the tumor?

\section{SUITABLE COMBINATION TARGETS: THE PI3K PATHWAY?}

Mitogen-activated protein kinase is not the only pathway to be constitutively active in melanoma (Figure 1). Another key pathway involved in cell survival, growth and apoptosis suppression is the PI3K/AKT pathway. Melanomas generally lack PI3K and $A K T$ mutations, but PTEN is lost in $30 \%$ of cell lines and $\sim 10 \%$ of clinical melanoma specimens. Recent studies have shown that AKT is able to transform melanocytes under hypoxic conditions (Bedogni et al, 2005), and there is evidence of cooperation between BRAF V600E and AKT in melanoma development (Cheung et al, 2008). Of the three AKT family members (AKT13 ), about $50 \%$ of melanoma cells have constitutive activity in AKT3 (Stahl et al, 2004). Inhibition of AKT activity in melanoma, using either PI3K inhibitors or selective RNAi to AKT3, or both, reduces growth and induces some degree of apoptosis (Stahl et al, 2004). There is preclinical evidence to suggest that there is a synergistic anti-melanoma activity when MEK and PI3K/AKT are inhibited concomitantly. Thus, the topical application of U0126 and LY294002 led to the regression of xenografts in a TPRas-induced melanoma model (Bedogni et al, 2004) associated with increased apoptosis and a reduced angiogenic response. Other studies have shown that PI3K and MEK inhibitors synergise to reduce growth and survival of melanoma cell lines grown under 3D organotypic cell culture conditions (Smalley et al, 2006; Meier et al, 2007). There is also evidence that siRNA knockdown of AKT3 and BRAF V600E leads to the enhanced inhibition of melanoma xenograft growth in nude mice (Cheung et al, 2008; Tran et al, 2008). 


\section{SUITABLE COMBINATION TARGETS: mTOR SIGNALLING?}

The mammalian target of rapamycin (mTOR) is a kinase that occupies a pivotal position in growth factor receptor and nutrient availability signalling, and has important downstream effects on cell growth and survival. It is also a part of the AKT signalling pathway and regulates the activity of this kinase in complex ways. Mammalian target of rapamycin can either positively or negatively regulate AKT signalling, and this is dependent on the composition of the mTOR signalling complex. Thus, mTOR forms at least two protein signalling complexes called mTORC1 and mTORC2. mTORC1 consists of mTOR, as well as the regulatory proteins raptor and mLST8. Activation of the mTORC1 signalling complex leads to the phosphorylation and activation of p70 S6kinase (S6K) and the eukaryotic initiation factor 4E-binding protein (4EBP1) (Reiling and Sabatini, 2006). In contrast, the mTORC2 complex also consists of mTOR and mLST8, and additionally includes the regulatory proteins rictor and $\mathrm{mSin}-1$. The differences between the two mTOR complexes are highly relevant, as an active mTORC1 complex suppresses AKT signalling, whereas mTORC2 stimulates AKT signalling through a phosphorylation event. A number of small molecule inhibitors are available that target mTORC1 but not mTORC2 signalling. The best characterised of these is rapamycin, a drug which is currently FDA-approved for use as an immunosuppressive agent after organ transplantation. There is evidence from the literature that mTOR signalling is active in melanoma. Studies have shown the presence of constitutive phospho-S6K in a high proportion of metastatic melanoma samples (Karbowniczek et al, 2008). Further work has shown that rapamycin has growth inhibitory effects across a panel of human melanoma cell lines (Molhoek et al, 2005), and there is evidence of synergistic pro-apoptotic activity between sorafenib/MEK inhibitors and rapamycin in preclinical models of melanoma (Molhoek et al, 2005; Meier et al, 2007; Lasithiotakis et al, 2008). Mechanistically, it seems that combined MEK/mTOR inhibition reduces the expressions of $\mathrm{Mcl}-1$ and $\mathrm{Bcl}-2$, two proteins that are known to suppress apoptosis induction in melanoma cells (Lasithiotakis et al, 2008).

\section{SUITABLE COMBINATION TARGETS: CHEMOTHERAPY}

Although most attention preclinically is focused on the combined inhibition of signal transduction pathways, most clinical interest is focused on the combination of targeted therapy agents with chemotherapy. Already, there are early suggestions that sorafenib may enhance the activity of carboplatin/paclitaxel and dacarbazine (McDermott et al, 2008). From a biochemical standpoint, very little is known about how MAPK signalling may modulate responses to chemotherapy, although it is known that constitutive MAPK activity, arising from mutations in HRAS, mediates cisplatin resistance through an increased DNA repair activity (Yen et al, 1997). There is evidence for the role of MEK in the upregulation of the DNA repair genes XRCC1 and ERCC1 after DNA damage and that this may be mediated by the transcription factor GATA-1 (Andrieux et al, 2007).

Rather more is known about the interaction between MEK inhibitors and the microtubule-stabilising taxanes. Treatment of cancer cells with paclitaxel is known to activate the MAPK pathway, and inhibition of MEK in combination with paclitaxel leads to additive effects on cell growth inhibition and apoptosis induction. It has also been shown that in addition to its effect on the G1/S-phase cell cycle transition, MAPK activity is also critical during meiosis and mitosis. Activated MAPK components (including BRAF) localise to the asters and kinetochores during mitosis, as well as to the actin-microtubule cytoskeleton. Earlier studies have shown that the orally available MEK inhibitor CI-1040 enhances the efficacy of paclitaxel in lung cancer xenograft models (McDaid et al, 2005), and that the MEK inhibitor AZD6244 enhances the pro-apoptotic effects of docetaxel in human melanoma cells (Haass et al, 2008). There is some suggestion that this combination may be effective because MEK activity is required for successful execution of mitosis, and the co-administration of an MEK inhibitor and paclitaxel enhances the level of mitotic catastrophe. These combinations are now undergoing evaluation for a number of solid tumors in phase I clinical trials, and a randomised phase II trial comparing AZD6244 combined with docetaxel, with docetaxel alone is planned.

\section{CLINICAL STUDIES ON BRAF/MEK INHIBITORS}

The current expectation is that BRAF inhibitors will be used in combination with either other targeted therapy agents or established chemotherapy regimens. However, it is necessary to rigorously evaluate each of the novel BRAF and MEK inhibitors as single agents so that we understand their distinct pharmacological properties and their respective abilities to hit their target and perturb proliferation or cell viability. Only with this knowledge can we make an informed selection of agents for further development.

Sorafenib is the most thoroughly investigated signal transduction inhibitor in clinical trials of melanoma patients. As a single agent, it was associated with only one objective response among 61 patients (Eisen et al, 2006). A small subset of patients with previously progressive metastatic disease maintained stable disease for more than 6 months, but an insufficient number to support the inclusion of sorafenib into a randomised phase III trial as a single agent. Analysis of patient samples pre- and posttreatment revealed that sorafenib incompletely inhibited ERK phosphorylation. This observation may be significant as preclinical studies show that complete pathway inhibition is needed to induce cell cycle arrest (Solit et al, 2006; Haass et al, 2008). In the single-agent phase II trials with sorafenib, the common toxicities were hypertension and hand-foot syndrome, consistent with the toxicities described with inhibitors of VEGF signalling. On the basis of these observations, the inhibitory activity of sorafenib against VEGF receptors (VEGFR2, VEGFR3 and VEGFR1) was explored with dynamic contrast-enhanced MRI and the results supported such an effect.

The combination of sorafenib with either carboplatin/paclitaxel or temozolomide has suggested some benefits. Consistent with the hypothesis that sorafenib is a weak inhibitor of BRAF in humans, an association between BRAF mutation and benefit from sorafenib/chemotherapy regimens has not been observed (Flaherty et al, 2008). A small, randomised phase II trial suggested benefit when sorafenib was added to dacarbazine compared with dacarbazine alone (McDermott et al, 2008). A phase III trial comparing sorafenib combined with chemotherapy with chemotherapy alone in patients with melanoma has completed accrual and results are expected in late 2009. The mechanism by which sorafenib appears to enhance the cytotoxicity of chemotherapy remains in question, and is complicated by the broadspectrum anti-kinase activity of sorafenib. Although there is evidence that MEK inhibition may sensitise cells to certain chemotherapies, it is not clear whether this mechanism is shared by sorafenib. A number of other studies have shown that inhibitors of VEGF signalling, such as bevacizumab, synergise with chemotherapy drugs, suggesting that the anti-VEGF activity of sorafenib may underlie its interaction with chemotherapy (Folkins et al, 2007). The broad-spectrum effects of sorafenib also leave open the possibility that anti-tumor immunity may be impaired after treatment (Hipp et al, 2008). As partial MAP kinase pathway inhibition, angiogenesis inhibition and suppression of anti-tumor response might combine to be effective in some patients, but counter-productive in others, significant additional work remains 
to be done to elucidate which patients may benefit from a sorafenib/chemotherapy approach. Some clinical studies have suggested that the presence of BRAF mutations confers a lower likelihood of response or disease control with multi-agent chemotherapy regimens (Kumar et al, 2003). This is clearly an area deserving further investigation, as it is unclear whether inhibition of BRAF would reverse this resistance, or whether that property is mediated through other pathways.

The MEK inhibitors-PD0325901 and AZD6244-have also progressed through phase I, and in the case of AZD6244 phase II clinical trials. PD0325901 has been evaluated in a phase I trial, in which most of the patients had melanoma. Of these patients, some experienced an objective response ( 2 out of 27) and 5 additional patients showed some disease stabilisation. Dose-limiting diarrhoea and rash precluded further dose escalation and possibly preclude the delivery of a dose required to adequately suppress the MAPK pathway. Phase II trials of this drug were suspended because of the occurrence of retinal vein thrombosis in several patients. AZD6244 was evaluated in a phase I trial among patients with advanced solid tumors (Adjei et al, 2008). Of the melanoma patients treated, none had an objective response; however, four patients maintained disease stabilisation for more than 6 months, suggesting a clinical benefit. A randomised phase II trial was completed among 200 patients with melanoma. Patients were randomised in a 1:1 manner to AZD6244 or temozolomide (Dummer et al, 2008). Five objective responses were observed among 42 patients with $B R A F$ V600E mutations (12\% objective response rate), indicating that a subset of $B R A F$ mutant melanomas may be sensitive to this agent. The trial was designed to detect an improvement in progression-free survival compared with single-agent temozolomide. As this activity was not seen, AZD6244 was deemed insufficient to warrant further single-agent clinical trials in melanoma.

As the preclinical data support the selective activity of RAF and MEK inhibitors in BRAF mutant melanoma, it is logical to accrue patients to phase II trials with these agents. This requires the elaboration of real-time mutation screening for inclusion into clinical trials, a hurdle not previously surmounted in earlier targeted therapy trials in cancer. The identification of concomitant genetic alterations or other markers of aberrant signal transduction in the same tumor samples used for BRAF mutation testing will set the foundation for exploration of markers of response or resistance. If a responsive subset of $B R A F$-mutated melanomas can be identified, it may be possible to rapidly develop RAF and MEK inhibitors as single agents for this population, whereas combination strategies are explored for the remaining.

Although the preclinical data are compelling in support of combining BRAF inhibition with a PI3K/AKT or mTOR inhibitor, the availability of agents targeting the mediators for phase I combination trials is severely limited. Potent and selective PI3K or AKT inhibitors are currently used in phase I clinical trials. As they emerge from phase I trials, there is the possibility of evaluating rational combinations with selective RAF or MEK inhibitors. Only rapamycin-analog mTOR inhibitors are readily available. It is not clear that these agents adequately block the upstream activation of this pathway, as they only block TORC1 complex signalling and permit enhanced activation of AKT by TORC2. A phase II trial was conducted with single-agent temsirolimus in 33 patients with metastatic melanoma unselected for any molecular or genetic features (Margolin et al, 2005). One partial response was observed and median progression-free survival was just over 2 months, consistent with an inactive therapy. However, there was little reason to hope that this therapy would have a stand-alone role. Rapamycin analogs are sufficiently tolerable to be evaluated in combination with other agents, and are presently the only agents one can feasibly combine with emerging BRAF or MEK inhibitors.

\section{CONCLUSION}

Although MAPK activation is a key step in the oncogenic transformation of melanocytes into melanoma, it appears that only a small minority of patients will benefit from the single-agent BRAF/MEK inhibitor treatment. The redundancy within the multiple signalling pathways activated in melanoma, along with the likelihood of drug resistance, suggests that combination therapy strategies will be required for effective disease management. It is further likely that the same molecular pathways involved in melanoma progression will also play important roles in both the angiogenic and immune response of melanoma, so that the targeting of these molecular pathways may have other beneficial effects beyond growth inhibition. As melanomas are more genetically heterogeneous than first thought, it is likely that 'personalised' combination therapies will need to be developed, whereby patient therapy is dictated by the constellation of mutations found within their tumors. The idea of personalised cancer therapy has already been demonstrated by the use of epidermal growth factor receptor inhibitors in lung cancer, where only $\sim 10 \%$ of patients harbour epidermal growth factor mutations and most fit a particular profile (adenocarcinoma, never-smoker, East-Asian ethnic origin and female). Similarly, in melanoma, there are early reports of success when selected groups of patients harbouring activating mutations in the receptor tyrosine kinase $c$-KIT have been treated with imatinib (Hodi et al, 2008). Clearly, there is much still to do, but we believe that targeting the correct combinations of signalling pathways in carefully selected groups of patients could give the therapeutic breakthrough that has been long overdue in melanoma.

\section{REFERENCES}

Adjei AA, Cohen RB, Franklin W, Morris C, Wilson D, Molina JR, Hanson LJ, Gore L, Chow L, Leong S, Maloney L, Gordon G, Simmons H, Marlow A, Litwiler K, Brown S, Poch G, Kane K, Haney J, Eckhardt SG (2008) Phase I pharmacokinetic and pharmacodynamic study of the oral, smallmolecule mitogen-activated protein kinase kinase 1/2 inhibitor AZD6244 (ARRY-142886) in patients with advanced cancers. J Clin Oncol 26: $2139-2146$

Andrieux LO, Fautrel A, Bessard A, Guillouzo A, Baffet G, Langouet S (2007) GATA-1 is essential in EGF-mediated induction of nucleotide excision repair activity and ERCC1 expression through ERK2 in human hepatoma cells. Cancer Res 67: 2114-2123

Bedogni B, O'Neill MS, Welford SM, Bouley DM, Giaccia AJ, Denko NC, Powell MB (2004) Topical treatment with inhibitors of the phosphatidylinositol $3^{\prime}$-kinase/Akt and Raf/mitogen-activated protein kinase kinase/extracellular signal-regulated kinase pathways reduces melanoma

development in severe combined immunodeficient mice. Cancer Res 64 $2552-2560$

Bedogni B, Welford SM, Cassarino DS, Nickoloff BJ, Giaccia AJ, Powell MB (2005) The hypoxic microenvironment of the skin contributes to Akt-mediated melanocyte transformation. Cancer Cell 8: 443-454

Cheung M, Sharma A, Madhunapantula SV, Robertson GP (2008) Akt3 and mutant V600E B-Raf cooperate to promote early melanoma development. Cancer Res 68: 3429-3439

Davies H, Bignell GR, Cox C, Stephens P, Edkins S, Clegg S, Teague J, Woffendin H, Garnett MJ, Bottomley W, Davis N, Dicks E, Ewing R, Floyd Y, Gray K, Hall S, Hawes R, Hughes J, Kosmidou V, Menzies A, Mould C, Parker A, Stevens C, Watt S, Hooper S, Wilson R, Jayatilake H, Gusterson BA, Cooper C, Shipley J, Hargrave D, Pritchard-Jones K, Maitland N, Chenevix-Trench G, Riggins GJ, Bigner DD, Palmieri G, Cossu A, Flanagan A, Nicholson A, Ho JW, Leung SY, Yuen ST, 
Weber BL, Seigler HF, Darrow TL, Paterson H, Marais R, Marshall CJ, Wooster R, Stratton MR, Futreal PA (2002) Mutations of the BRAF gene in human cancer. Nature 417: 949-954

Dummer R, Robert C, Chapman PB, Sosman JA, Middleton MR, Bastholt L, Kemsley MV, Cantarini MV, Morris C, Kirkwood JM (2008) AZD6244 (ARRY-142886) vs temozolomide (TMZ) in patients with advanced melanoma: An open-label, randomized, multicenter, phase II study. J Clin Oncol 26, abstract 9033

Eisen T, Ahmad T, Flaherty KT, Gore M, Kaye S, Marais R, Gibbens I, Hackett S, James M, Schuchter LM, Nathanson KL, Xia C, Simantov R, Schwartz B, Poulin-Costello M, O'Dwyer PJ, Ratain MJ (2006) Sorafenib in advanced melanoma: a Phase II randomised discontinuation trial analysis. Br J Cancer 95: $581-586$

Flaherty KT, Schiller J, Schuchter LM, Liu G, Tuveson DA, Redlinger M, Lathia C, Xia C, Petrenciuc O, Hingorani SR, Jacobetz MA, Van Belle PA, Elder D, Brose MS, Weber BL, Albertini MR, O’Dwyer PJ (2008) A phase I trial of the oral, multikinase inhibitor sorafenib in combination with carboplatin and paclitaxel. Clin Cancer Res 14: 4836-4842

Folkins C, Man S, Xu P, Shaked Y, Hicklin DJ, Kerbel RS (2007) Anticancer therapies combining antiangiogenic and tumor cell cytotoxic effects reduce the tumor stem-like cell fraction in glioma xenograft tumors. Cancer Res 67: 3560 - 3564

Gray-Schopfer V, Wellbrock C, Marais R (2007) Melanoma biology and new targeted therapy. Nature 445: $851-857$

Haass NK, Sproesser K, Nguyen TK, Contractor R, Medina CA, Nathanson KL, Herlyn M, Smalley KS (2008) The mitogen-activated protein/ extracellular signal-regulated kinase kinase inhibitor AZD6244 (ARRY142886) induces growth arrest in melanoma cells and tumor regression when combined with docetaxel. Clin Cancer Res 14: 230-239

Hingorani SR, Jacobetz MA, Robertson GP, Herlyn M, Tuveson DA (2003) Suppression of BRAF(V599E) in human melanoma abrogates transformation. Cancer Research 63: 5198-5202

Hipp MM, Hilf N, Walter S, Werth D, Brauer KM, Radsak MP, Weinschenk T, Singh-Jasuja H, Brossart P (2008) Sorafenib, but not sunitinib, affects function of dendritic cells and induction of primary immune responses. Blood 111: $5610-5620$

Hodi FS, Friedlander P, Corless CL, Heinrich MC, Mac Rae S, Kruse A, Jagannathan J, Van den Abbeele AD, Velazquez EF, Demetri GD, Fisher $\mathrm{DE}$ (2008) Major response to imatinib mesylate in KIT-mutated melanoma. J Clin Oncol 26: 2046-2051

Houben R, Vetter-Kauczok CS, Ortmann S, Rapp UR, Broecker EB, Becker JC (2008) Phospho-ERK staining is a poor indicator of the mutational status of BRAF and NRAS in human melanoma. J Invest Dermatol 128: $2003-2012$

Karbowniczek M, Spittle CS, Morrison T, Wu H, Henske EP (2008) mTOR is activated in the majority of malignant melanomas. J Invest Dermatol 128: $980-987$

King AJ, Patrick DR, Batorsky RS, Ho ML, Do HT, Zhang SY, Kumar R, Rusnak DW, Takle AK, Wilson DM, Hugger E, Wang L, Karreth F, Lougheed JC, Lee J, Chau D, Stout TJ, May EW, Rominger CM, Schaber MD, Luo L, Lakdawala AS, Adams JL, Contractor RG, Smalley KS, Herlyn M, Morrissey MM, Tuveson DA, Huang PS (2006) Demonstration of a genetic therapeutic index for tumors expressing oncogenic BRAF by the kinase inhibitor SB-590885. Cancer Res 66: 11100-11105

Kumar R, Angelini S, Czene K, Sauroja I, Hahka-Kemppinen M, Pyrhonen S, Hemminki K (2003) BRAF mutations in metastatic melanoma: a possible association with clinical outcome. Clin Cancer Res 9: 3362-3368

Lasithiotakis KG, Sinnberg TW, Schittek B, Flaherty KT, Kulms D, Maczey E, Garbe C, Meier FE (2008) Combined inhibition of MAPK and mTOR signaling inhibits growth, induces cell death, and abrogates invasive growth of melanoma cells. J Invest Dermatol 128: 2013-2023

Leboeuf R, Baumgartner JE, Benezra M, Malaguarnera R, Solit D, Pratilas CA, Rosen N, Knauf JA, Fagin JA (2008) BRAFV600E mutation is associated with preferential sensitivity to mitogen-activated protein kinase kinase inhibition in thyroid cancer cell lines. The Journal of Clinical Endocrinology and Metabolism 93: 2194-2201

Margolin K, Longmate J, Baratta T, Synold T, Christensen S, Weber J, Gajewski T, Quirt I, Doroshow JH (2005) CCI-779 in metastatic melanoma: a phase II trial of the California Cancer Consortium. Cancer 104: $1045-1048$

McDaid HM, Lopez-Barcons L, Grossman A, Lia M, Keller S, Perez-Soler R, Horwitz SB (2005) Enhancement of the therapeutic efficacy of taxol by the mitogen-activated protein kinase kinase inhibitor CI-1040 in nude mice bearing human heterotransplants. Cancer Res 65: 2854-2860

McDermott DF, Sosman JA, Gonzalez R, Hodi FS, Linette GP, Richards J, Jakub JW, Beeram M, Tarantolo S, Agarwala S, Frenette G, Puzanov I, Cranmer L, Lewis K, Kirkwood J, White JM, Xia C, Patel K, Hersh E (2008) Double-blind randomized phase II study of the combination of sorafenib and dacarbazine in patients with advanced melanoma: a report from the 11715 Study Group. J Clin Oncol 26: 2178-2185

Meier F, Busch S, Lasithiotakis K, Kulms D, Garbe C, Maczey E, Herlyn M, Schittek B (2007) Combined targeting of MAPK and AKT signalling pathways is a promising strategy for melanoma treatment. Br J Dermatol 156: $1204-1213$

Molhoek KR, Brautigan DL, Slingluff Jr CL (2005) Synergistic inhibition of human melanoma proliferation by combination treatment with B-Raf inhibitor BAY43-9006 and mTOR inhibitor Rapamycin. J Transl Med 3: 39

Montagut C, Sharma SV, Shioda T, McDermott U, Ulman M, Ulkus LE, Dias-Santagata D, Stubbs H, Lee DY, Singh A, Drew L, Haber DA, Settleman J (2008) Elevated CRAF as a potential mechanism of acquired resistance to BRAF inhibition in melanoma. Cancer Res 68: $4853-4861$

Reiling JH, Sabatini DM (2006) Stress and mTORture signaling. Oncogene 25: $6373-6383$

Satyamoorthy K, Li G, Gerrero MR, Brose MS, Volpe P, Weber BL, van Belle P, Elder DE, Herlyn M (2003) Constitutive mitogen-activated protein kinase activation in melanoma is mediated by both BRAF mutations and autocrine growth factor stimulation. Cancer Research 63: 756-759

Sharma A, Trivedi NR, Zimmerman MA, Tuveson DA, Smith CD, Robertson GP (2005) Mutant V599EB-Raf regulates growth and vascular development of malignant melanoma tumors. Cancer Res 65: 2412-2421

Smalley KS, Haass NK, Brafford PA, Lioni M, Flaherty KT, Herlyn M (2006) Multiple signaling pathways must be targeted to overcome drug resistance in cell lines derived from melanoma metastases. Mol Cancer Ther 5: 1136-1144

Smalley KS, Lioni M, Palma MD, Xiao M, Desai B, Egyhazi S, Hansson J, Wu H, King AJ, Van Belle P, Elder DE, Flaherty KT, Herlyn M, Nathanson KL (2008) Increased cyclin D1 expression can mediate BRAF inhibitor resistance in BRAF V600E-mutated melanomas. Mol Cancer Ther 7: $2876-2883$

Solit DB, Garraway LA, Pratilas CA, Sawai A, Getz G, Basso A, Ye Q, Lobo JM, She Y, Osman I, Golub TR, Sebolt-Leopold J, Sellers WR, Rosen N (2006) BRAF mutation predicts sensitivity to MEK inhibition. Nature 439: $358-362$

Stahl JM, Sharma A, Cheung M, Zimmerman M, Cheng JQ, Bosenberg MW, Kester M, Sandirasegarane L, Robertson GP (2004) Deregulated Akt3 activity promotes development of malignant melanoma. Cancer Res 64: $7002-7010$

Tran MA, Gowda R, Sharma A, Park EJ, Adair J, Kester M, Smith NB, Robertson GP (2008) Targeting V600EB-Raf and Akt3 using nanoliposomal-small interfering RNA inhibits cutaneous melanocytic lesion development. Cancer Res 68: $7638-7649$

Tsai J, Lee JT, Wang W, Zhang J, Cho H, Mamo S, Bremer R, Gillette S, Kong J, Haass NK, Sproesser K, Li L, Smalley KS, Fong D, Zhu YL, Marimuthu A, Nguyen H, Lam B, Liu J, Cheung I, Rice J, Suzuki Y, Luu C, Settachatgul C, Shellooe R, Cantwell J, Kim SH, Schlessinger J, Zhang KY, West BL, Powell B, Habets G, Zhang C, Ibrahim PN, Hirth P, Artis DR, Herlyn M, Bollag G (2008) Discovery of a selective inhibitor of oncogenic B-Raf kinase with potent antimelanoma activity. Proc Natl Acad Sci USA 105: 3041 - 3046

Verhaegen M, Bauer JA, Martin de la Vega C, Wang G, Wolter KG, Brenner JC, Nikolovska-Coleska Z, Bengtson A, Nair R, Elder JT, Van Brocklin M, Carey TE, Bradford CR, Wang S, Soengas MS (2006) A novel BH3 mimetic reveals a mitogen-activated protein kinase-dependent mechanism of melanoma cell death controlled by p53 and reactive oxygen species. Cancer Res 66: 11348-11359

Wang Y, Van Becelaere K, Jiang P, Przybranowski S, Omer C, SeboltLeopold J (2005) A role for K-ras in conferring resistance to the MEK inhibitor, CI-1040. Neoplasia 7: 336-347

Yen L, Nie ZR, You XL, Richard S, Langton-Webster BC, Alaoui-Jamali MA (1997) Regulation of cellular response to cisplatin-induced DNA damage and DNA repair in cells overexpressing p185(erbB-2) is dependent on the ras signaling pathway. Oncogene 14: 1827-1835 